\title{
Role of EGF inhibitors in the treatment of recurrent or metastatic squamous cell head and neck cancer
}

This article was published in the following Dove Press journal:

Cancer Management and Research

2 November 2009

Number of times this article has been viewed

Jochen H Lorch

Dana Farber Cancer Institute, Boston, MA, USA
Correspondence: Jochen Lorch Dana Farber Cancer Institute, SW430, 44 Binney Street, Boston, MA 02I I5, USA Email jochen_lorch@dfci.harvard.edu

\begin{abstract}
Squamous cell cancer of the head and neck (SCCHN) is a major contributor to morbidity and mortality worldwide. In recent years, inhibition of the epidermal growth factor receptor has become an established treatment strategy in SCCHN both in the up-front treatment and in the recurrent and metastatic setting. This review summarizes the most important developments of the recent past and provides an overview of newer developments.
\end{abstract}

Keywords: squamous cell cancer, head, neck, epidermal growth factor receptor

\section{Background}

Squamous cell cancer of the head and neck (SCCHN) is a major contributor to morbidity and mortality and accounts for approximately 5\% of all new cancer diagnoses. Worldwide, more than 600,000 new cases are diagnosed every year and 350,000 cancer deaths can be attributed to this disease. ${ }^{1}$ The majority of patients are diagnosed in the locally advanced and metastatic stages and survival has been poor despite the fact that $\mathrm{SCCHN}$ is very sensitive to treatment with a high rate of remissions.

As chemotherapy combinations have become increasingly intense in recent years, it has become evident that a limit has been reached where gains in activity and response rate are offset by greater toxicities. ${ }^{2}$ Targeted therapies with their different side effect profile offer the possibility to further expand and intensify combination therapy and numerous trials have explored this strategy in many fields of solid tumor oncology including head and neck cancer. The advances in the basic sciences and an ever increasing number of potential targets have created a new wealth of therapeutic possibilities that are now explored in various stages of pre-clinical and clinical development. In the last few years in particular, progress has been made. However, an increasingly comprehensive picture is just beginning to emerge and clinical testing remains critical.

Head and neck cancer treatment is also transforming as a result of targeted therapies. Among the first potential targets that were recognized in SCCHN was the epidermal growth factor receptor (EGFR). It is overexpressed in many types of epithelial neoplasms including SCCHN where robust overexpression is found in $>90 \%$ of cases. The EGFR/ErbB1 receptor is a member of the tyrosine kinase superfamily along with the other three members of the erbB tyrosine kinase family which include erbB2 (HER2/neu), erbB3 (HER3), and erbB4 (HER4). ${ }^{3}$

The EGFR includes a ligand binding extracellular domain at the N-termial end, a transmembrane section that anchors the molecule in the cell membrane and an 
intracellular domain that transmits signals to a multitude of downstream signaling pathways. Upon binding of a ligand such as EGF or transforming growth factor- $\beta$ (TGF- $\beta$ ), dimerization of the EGFR occurs and phosphorylation of intracellular tyrosine residues trigger activation of the EGFR complex and the downstream signaling cascade. ${ }^{4}$ Among the pathways involved in EGFR signal transmission are the MAP kinase and the PI3 kinase pathways with their downstream effectors, respectively, that control a vast array of intracellular processes including cell cycling, cell adhesion and migration among others..$^{5-8}$

Strategies to block activity of the EGFR have been explored in the past years and have become an established part of modern SCCHN treatment. Inhibition of EGFR activity can be achieved through the following main methods: 1) through antibody binding to the receptor, which interferes with ligand binding and induces internalization and subsequent breakdown of the antibody-EGFR complex in addition to immunologic events that may result in destabilization of targeted cells, 2) with small molecules that act as competitive inhibitors to the intracellular adenosine triphosphate (ATP)-binding site, and 3) through specific downregulation of the EGFR.

\section{EGFR antibodies}

Anti-EGFR chimeric monoclonal antibodies include cetuximab $\left(\mathrm{C} 225\right.$, Erbitux $\left.^{\circledR}\right)$ and fully humanized antibodies such as panitumumab, matuzumab, nimotuzumab, and zalutumumab. They are specific to the extracellular portion of the EGFR and exert their anti-EGFR activity by inhibiting ligand binding, thus interfering with EGFR activation. They also induce internalization of the EGFR resulting in a lower number of EGFR at the cell surface. Furthermore, antibody binding may trigger an immunologic response resulting in the de-stabilization and ultimately apoptosis of the cancer cell.

Among this class of drugs, cetuximab (Erbitux ${ }^{\circledR}$ ) has the longest track record and has been studied extensively in head and neck cancer. In patients with locally advanced, potentially curable SCCHN, data from a randomized trial shows that the addition of cetuximab to radiation in the definitive treatment of locally advanced SCCHN results in improved local control, overall survival and time to progression compared with radiation alone. ${ }^{9}$ In a landmark study in 424 patients with stage III and IV squamous cell cancer of the oropharynx, hypopharynx, or larynx, the median duration of locoregional control was 24.4 months among patients treated with cetuximab plus radiotherapy and 14.9 months among those given radiotherapy (hazard ratio for locoregional progression or death, $0.68 ; P=0.005)$. With a median follow-up of 54 months, the median duration of overall survival was 49 months among patients treated with combined therapy and 29.3 months among those treated with radiotherapy alone (hazard ratio for death, $0.74 ; P=0.03$ ) and progression-free survival was increased significantly as well. Interestingly, patients with oropharyngeal primary tumors enjoyed the greatest benefit compared to other locations highlighting biological differences within SCCHN, which affect response to treatment and outcomes.

When cetuximab is used as the single agent in metastatic disease SCCHN patients, the response rate is modest around $15 \%$. However, a large, randomized trial tested CDDP/5FU with or without the addition of cetuximab in 442 patients with overall survival as the primary endpoint. Median survival was 7.4 months in the chemotherapy alone arm compared to 10.1 months for chemotherapy plus cetuximab $\operatorname{arm}(P=0.036)$ which was one of the longest median survival times in this patient group ever recorded. ${ }^{10}$ Side effects typically include the acneiforme rash ( $80 \%$ overall, $3 \%$ severe) and interestingly correlates with a tumor response. In contrast, EGFR expression as measured by immunohistochemistry in this study was not a reliable predictor of tumor response. ${ }^{11}$ Allergic reactions are found in approximately $3 \%$ of patients who receive cetuximab and recent evidence suggests that reactions at least in some regions of the United States are triggered by carbohydrate residues added during the manufacturing process. ${ }^{12}$

Other studies are evaluating cetuximab in combination with paclitaxel or docetaxel and promising response rates including a significant number of complete responses have been reported as abstracts. ${ }^{13}$ A particularly interesting drug combination is based on the observation that up-regulation of vascular endothelial growth factor (VEGF) has been associated with cetuximab resistance, raising the possibility that combined targeting of VEGF and EGFR may enhance antitumor activity. The results of a phase II trial using cetuximab in combination with the humanized monoclonal VEGF antibody bevacizumab in 28 patients with incurable head and neck cancer showed a partial response in $20 \%{ }^{14}$ Perhaps more importantly this limited analysis also showed that the combination was well tolerated and appeared safe with no cases of grade 4 bleeding, the most concerning side effect given the risk of severe and sometimes fatal bleeding in squamous cell cancer of the lung in patients treated with bevacizumab. Studies using a similar, but fully humanized monoclonal anti-EGFR antibodies such as panitumumab are also under way but no data from phase II trials in patients 
with metastatic disease has yet been published. Other ongoing trials with cetuximab include a combination with a humanized monoclonal insulin-like growth factor receptor (IGFR) antibody IMC A12. Preclinical studies suggest synergy between EGF and IGFR inhibition and a high rate of cell death was observed. This trial is currently accruing patients to explore activity in incurable recurrent or metastatic disease patients and no results are available to date.

Other antibody based strategies using toxin-bound antibodies directed against the EGFR are currently being considered for use in head and neck cancer patients but no results are available to date.

\section{EGFR inhibition with small molecule tyrosine kinase inhibitors}

A number of EGFR-specific agents from the class of the small molecule tyrosine kinase inhibitors have emerged and are used in a variety of tumors, including SCCHN. They are competitive antagonists of the ATP-binding site, inhibiting phosphorylation and subsequently activation of the EGFR receptor. When used as single agent, the response rate appears comparable to that seen with anti-EGFR antibodies. Studies led by investigators at the University of Chicago evaluated gefitinib (Iressa ${ }^{\circledR}$ ) in patients with recurrent SCCHN. Fifty-two patients were treated with $500 \mathrm{mg}$ gefitinib per day and approximately half of the patients received gefitinib as second-line therapy. An objective response was found in $10.6 \%$ and - perhaps more interestingly - the disease control rate was 53\%. There appears to be a dose dependence as the response rate to gefitinib at a lower dose ( $250 \mathrm{mg}$ per day) induces responses is only $1.4 \% .{ }^{15}$ A similar agent, erlotinib (Tarceva ${ }^{\circledR}$ ), been tested in patients with recurrent disease and the response rate was $4 \%$ with a progression-free survival and overall survival similar to that seen with gefitinib. ${ }^{16}$ There is some evidence from these trials that response to erlotinib may be more likely in patients with a high EGFR copy number indicative of EGFR amplification. ${ }^{17}$ The side effect profile of the small molecule tyrosine kinase inhibitors are remarkably similar to the EGFR antibodies and involve acneiform skin rash, diarrhea, and electrolyte disturbances including hypocalcemia and hypomagnesemia, which are thought to be directly linked to the blocking of the EGFR-dependent renal reabsorption. Combination with traditional chemotherapy agents has also been tested. Erlotinib combined with cisplatin and docetaxel demonstrated a response rate of $67 \%$ with $4 / 43$ patients achieving a complete response. Median overall survival was 11 months $(8.61,22.5,95 \%$ confidence interval) and progression-free survival was 6.01 months $(4.37,8.25)$. However, six patients had grade $3 / 4$ febrile neutropenia, four patients had grade $3 / 4$ dehydration, three patients had grade 3 diarrhea, and two patients had grade 3/4 gastrointestinal (GI) bleeding. ${ }^{18}$ Similar to the combination of cetuximab with bevacizumab mentioned above, erlotinib has also been combined with bevacizumab in a phase I/II trial in a total of 48 patients with metastatic SCCHN and improved efficacy compared with erlotinib alone was found. Seven patients had a response, with four showing a complete response. Median overall survival and progression-free survival were 7.1 months (95\% CI: 5.7-9.0) and 4.1 months (95\% CI: 2.8-4.4), respectively. The most common side effects of any grade were rash and diarrhea (41 and 16 of 48 patients, respectively) and serious bleeding of grade 3 or higher occurred in three cases. ${ }^{19}$

Other small molecule tyrosine kinase inhibitors that inhibit a broader range of tyrosine kinases such as vandetanib $\left(\mathrm{AZ6474}\right.$, Zactima $^{\circledR}$ ) are also tested in SCCHN. Vandetanib was primarily developed as an inhibitor of the VEGF receptor but also has significant EGFR-inhibiting properties as well. It is currently evaluated in combination with docetaxel and no results in SCCHN have been published.

Downregulation of the EGFR by direct injection of antisense DNA is also pursued at specialized centers. Data from a phase I trial suggests that this approach is well tolerated and tumor responses were seen. ${ }^{20}$ However, given the scarcity of data and the invasiveness of the therapy, this approach needs further validation before a broader application could be considered.

Drug combinations such as inhibitors of the toll-like receptor antagonist along with EGFR inhibitors have been tested in cell culture studies and it appears that there may be synergy when both pathways are blocked. ${ }^{21}$ However, no clinical studies are currently exploring this strategy in head and neck cancer patients.

\section{Summary and outlook}

EGFR antagonists have added an invaluable tool to the modern medical treatment of SCCHN. While perhaps the greatest gains have been made with combining EGFR inhibition with radiotherapy in the locally advanced, curable stages, anti-EGFR therapy has also become an established part of palliative treatment of SCCHN patients. Small molecule tyrosine kinase inhibitors also hold promise of advancing current treatments given their considerable biological activity and their convenient administration. 
Despite this progress, formidable challenges remain. The identification of tumor and patient characteristics that are linked to treatment response are still at early stages in SCCHN and future success will depend on a broad effort of clinicians, translational and basic scientists. A personalized approach to treatment with EGFR inhibitors in SCCHN and cancer treatment in general will be critical to achieve cost effectiveness and maximize clinical impact on patient's lives.

\section{Disclosures}

The authors report no conflicts of interest in this work.

\section{References}

1. Jemal A, Siegel R, Ward E, Murray T, Xu J, Thun MJ. Cancer statistics, 2007. CA Cancer J Clin. 2007;57:43-66.

2. Choong N, Vokes E. Expanding role of the medical oncologist in the management of head and neck cancer. CA Cancer J Clin. 2008; $58: 32-53$.

3. Schlessinger J. Cell signaling by receptor tyrosine kinases. Cell. 2000;103:211-225.

4. Mark H. Kirschbaum YY. The ErbB/HER family of receptor tyrosine kinases: A potential target for chemoprevention of epithelial neoplasms. J Cell Biochem. 2000;77:52-60.

5. Bunn PA Jr, Franklin W. Epidermal growth factor receptor expression, signal pathway, and inhibitors in non-small cell lung cancer. Semin Oncol. 2002;29:38-44.

6. Chen P, Xie H, Sekar MC, Gupta K, Wells A. Epidermal growth factor receptor-mediated cell motility: phospholipase $\mathrm{C}$ activity is required, but mitogen-activated protein kinase activity is not sufficient for induced cell movement. J Cell Biol. 1994;127:847-857.

7. Ellerbroek SM, Halbleib JM, Benavidez M, et al. Phosphatidylinositol 3-kinase activity in epidermal growth factor- stimulated matrix metalloproteinase-9 production and cell surface association. Cancer Res. 2001;61:1855-1861.

8. Lorch JH, Klessner J, Park JK, et al. Epidermal growth factor receptor inhibition promotes desmosome assembly and strengthens intercellular adhesion in squamous cell carcinoma cells. $J$ Biol Chem. 2004;279:37191-37200.

9. Bonner JA, Harari PM, Giralt J, et al. Radiotherapy plus cetuximab for squamous-cell carcinoma of the head and neck. $N$ Engl J Med. 2006;354:567-578.

10. Vermorken JB, Mesia R, Vega V, et al. Cetuximab extends survival of patients with recurrent or metastatic SCCHN when added to first line platinum based therapy - Results of a randomized phase III (Extreme) study [abstract]. Proc Am Soc Clin Oncol. 2007;25:6091.
11. Kies MS, Ghebremichael MS, Katz TL, Herbst RS, Youssoufian H, Burtness B. EGFR expression by immunohistochemistry (IHC) and response to chemotherapy and cetuximab in squamous cell carcinoma of the head and neck (SCCHN) [abstract]. Proc Am Soc Clin Oncol. 2007;25:6024.

12. Chung CH, Mirakhur B, Chan E, et al. Cetuximab-induced anaphylaxis and IgE specific for galactose-alpha-1,3-galactose. $N$ Engl $J$ Med. 2008;358:1109-1117.

13. Hitt R, Irigoyen A, Nuñez J, et al. Phase II study of combination cetuximab and weekly paclitaxel in patients with metastatic/recurrent squamous cell carcinoma of head and neck (SCCHN): Spanish Head and Neck Cancer Group (TTCC) [abstract]. Proc Am Soc Clin Oncol. 2007;25:6012.

14. Gibson MK, Kies MS SK, et al. Cetuximab (C) and bevacizumab (B) in patients with recurrent or metastatic head and neck squamous cell carcinoma: An updated report [abstract]. Proc Am Soc Clin Oncol. 2009;27:6049.

15. Cohen EE, Kane MA, List MA, et al. Phase II trial of gefitinib $250 \mathrm{mg}$ daily in patients with recurrent and/or metastatic squamous cell carcinoma of the head and neck. Clin Cancer Res. 2005;11:8418-8424.

16. Soulieres D, Senzer NN, Vokes EE, Hidalgo M, Agarwala SS, Siu LL. Multicenter phase II study of erlotinib, an oral epidermal growth factor receptor tyrosine kinase inhibitor, in patients with recurrent or metastatic squamous cell cancer of the head and neck. J Clin Oncol. 2004;22:77-85.

17. Agulnik M, da Cunha Santos G, Hedley D, et al. Predictive and pharmacodynamic biomarker studies in tumor and skin tissue samples of patients with recurrent or metastatic squamous cell carcinoma of the head and neck treated with erlotinib. J Clin Oncol. 2007;25:2184-2190.

18. Kim ES, Kies MS, Glisson BS, et al. Final results of a phase II study of erlotinib, docetaxel and cisplatin in patients with recurrent/ metastatic head and neck cancer [abstract]. Proc Am Soc Clin Oncol. 2007;25:6013.

19. Cohen EE, Davis DW, Karrison TG, et al. Erlotinib and bevacizumab in patients with recurrent or metastatic squamous-cell carcinoma of the head and neck: a phase I/II study. Lancet Oncol. 2009;10:247-257.

20. Lai SY, Lui VW, Koppikar PJ, et al. Intratumoral epidermal growth factor receptor (EGFR) antisense (AS) DNA in recurrent squamous cell carcinoma of the head and neck (SCCHN): A phase I trial [abstract]. Proc Am Soc Clin Oncol. 2007;25:6009.

21. Damiano V, Caputo R, Bianco R, et al. Novel toll-likereceptor 9 agonist induces epidermal growth factor receptor (EGFR) inhibition and synergistic antitumor activity with EGFR inhibitors. Clin Cancer Res. 2006;12:577-583.
Cancer Management and Research

\section{Publish your work in this journal}

Cancer Management and Research is an international, peer-reviewed open access journal focusing on cancer research and the optimal use of preventative and integrated treatment interventions to achieve improved outcomes, enhanced survival and quality of life for the cancer patient. The journal welcomes original research, clinical \& epidemiological

\section{Dovepress}

studies, reviews \& evaluations, guidelines, expert opinion \& commentary, case reports \& extended reports. The manuscript management system is completely online and includes a very quick and fair peerreview system, which is all easy to use. Visit http://www.dovepress.com/ testimonials.php to read real quotes from published authors. 